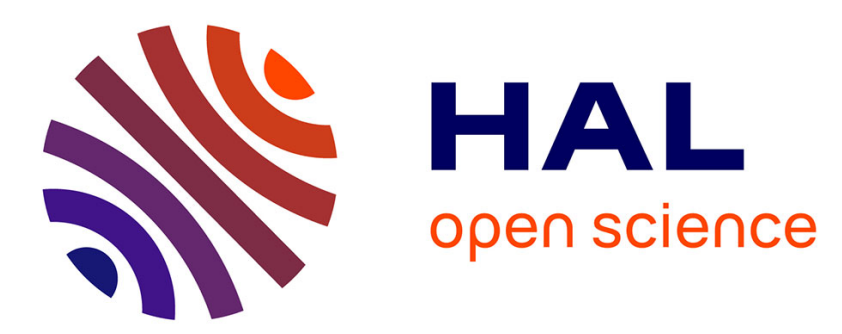

\title{
A-ECMS and SDP Energy Management Algorithms applied to a Fuel Cell Electric Scooter
}

Qi Jiang, Olivier Béthoux, Florence Ossart, Éric Berthelot, Claude Marchand

\section{To cite this version:}

Qi Jiang, Olivier Béthoux, Florence Ossart, Éric Berthelot, Claude Marchand. A-ECMS and SDP Energy Management Algorithms applied to a Fuel Cell Electric Scooter. Vehicle Power and Propulsion Conference (VPPC), Dec 2017, Belfort, France. pp.1-5, 10.1109/VPPC.2017.8330968 . hal-01693651

\section{HAL Id: hal-01693651 https://hal.science/hal-01693651}

Submitted on 11 Mar 2020

HAL is a multi-disciplinary open access archive for the deposit and dissemination of scientific research documents, whether they are published or not. The documents may come from teaching and research institutions in France or abroad, or from public or private research centers.
L'archive ouverte pluridisciplinaire HAL, est destinée au dépôt et à la diffusion de documents scientifiques de niveau recherche, publiés ou non, émanant des établissements d'enseignement et de recherche français ou étrangers, des laboratoires publics ou privés. 


\title{
A-ECMS and SDP Energy Management Algorithms applied to a Fuel Cell Electric Scooter
}

\author{
Qi JIANG, Olivier BETHOUX, Florence OSSART, Eric BERTHELOT, Claude MARCHAND \\ GeePs | Group of Electrical Engineering - Paris \\ UMR CNRS 8507, CentraleSupélec, Univ Paris-Sud, Sorbonne Universités, UPMC Univ Paris 06 \\ Gif-sur-Yvette, France \\ florence.ossart@geeps.centralesupelec.fr
}

\begin{abstract}
The energy management of a fuel cell system coupled to a supercapacitor energy storage system is studied, both in simulation and on a same-scale test bench. This system can be used to power an urban electric scooter. To obtain a low real-time hydrogen consumption, an online energy management strategy needs to be embedded. This paper assesses the best energy performance of two promising real-time energy management strategies for fuel cell electric vehicle applications: adaptive equivalent consumption minimization strategy and stochastic dynamic programming. For this purpose, a specific certification driving cycle is considered while an off-line algorithm is used as benchmark. Simulation experimental results show that SDP has a higher potential than A-ECMS, making SDP complexity worth handling.
\end{abstract}

Keywords-fuel cell electric vehicle; energy management; optimal control; adaptative equivalent consumption minimization strategy; Stochastic dynamic programming

\section{INTRODUCTION}

Fuel cell electric vehicles (FCEVs) are considered as a promising technology to build long range environment friendly vehicles [1]. FCEVs possess an electrochemical power source (the FC) and an energy storage system such as a supercapacitor (SC). This combination of two power sources is needed because the FC is too slow to fulfill the instantaneous need of traction power. In addition it improves the global system efficiency due to braking energy recovery and the opportunity to operate the FC around its best efficiency working points whatever the instantaneous power needed by the vehicle [2]. To achieve these two functionalities, a supervisor is needed in order to split the instantaneous power between the FC and the $\mathrm{SC}$ and minimize the total $\mathrm{H}_{2}$ consumption over a given driving cycle, while strictly meeting the driver's power demand and satisfying operating constraints.

Off-line optimization is used when the driving cycle is known, typically to perform system design, sizing and test in perfectly controlled conditions. Pontryagin's minimum principle (PMP) [3][4] and dynamic programming (DP) [5][6] are then widely used. They are hence very helpful for benchmarking studies.

In contrast, in real life conditions, the oncoming driving cycle cannot be foreseen and online methods are needed. The present paper focuses on two methods and applies them to a FC/SC system suitable for urban electric scooters. The system and its related real-time energy management strategies are evaluated both in simulation and on an experimental set-up. The first method, called adaptative equivalent consumption minimization strategy (A-ECMS) [7] is based on PMP. This approach is popular because of its simplicity, but it is also known for its possible unstable behavior. Hence, more complex approaches should be investigated. The second method under study is stochastic dynamic programming (SDP) [3], which generalizes DP in the case where random processes are involved, which is typically the case for real-life driving cycles. SDP is appealing because it has a clear theoretical background and allows to take into account statistical information about the driving cycle.

The paper objective is to assess the best potential of both online methods using a specific certification driving cycle. For this purpose, an off-line method is used as a reference, showing the minimum consumption achievable. The idea is to find out whether SDP complexity is worth handling with energy performance close to optimum. In this context, the paper is organized as follows. The FC/SC system and its model are described in Sections II. Off-line and online EMS are presented in Section III. In Section IV the model and test bench are first validated, then A-ECMS and SDP performances are compared to DP's. SDP appears to have a better potential.

\section{FC/SC SYSTEM DESCRIPTION AND MODELING}

This study deals with the hybrid FC/SC powertrain of a scooter. Fig. 1 shows the system architecture: the FC and the SC are connected via DC/DC converters to the DC bus which feeds the electric motor. The main power source of the system is a $1.2 \mathrm{~kW}$ proton exchange membrane FC, with a maximum efficiency for an output power around $300 \mathrm{~W}$. A bi-directional load allows to simulate the traction power needed by the scooter: during braking, power is sent back to the FC/SC system. The supervisor inputs are the SC voltage, used to estimate the SC state of charge, and the load power. These informations are used by the EMS to determine the instantaneous FC output power. Low level controlers manage the voltage and current in the different parts of the system.

The energy management algoritms require the system to be precisely modeled. The FC is modeled by its static V-I characteristic and its $H_{2}$ consumption curve $\dot{m}_{H_{2}}\left(P_{F C}\right)$. The SC stack is modeled as a RC circuit in parallel with an internal leakage current source with a quadratic dependence with 
respect to the external voltage. In a similar way, both DC/DC converters are modeled as ideal converters in parallel with leakage current sources to account for internal losses. The model parameters are identified by fitting experimental data.

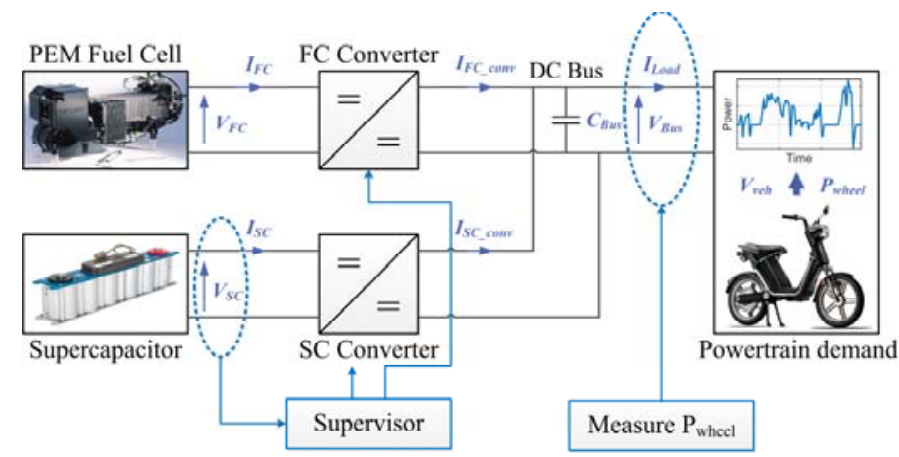

Fig. 1. FC/SC hybrid system under study and its supervisor

The power demand $P_{\text {wheel }}$ depends on the speed profile and is calculated using the motion equation (1):

$$
P_{\text {wheel }}=\left[0.5 \rho_{a i r} A C_{d} v^{2}+\mu_{r} m g+m a\right] . v
$$

where $\rho_{\text {air }}$ is density of air; $A$ is the reference area; $C_{d}$ is the drag coefficient; $\mu_{r}$ is the rolling resistance coefficient; $m$ is the vehicle mass; $g$ is the gravitational acceleration; $v$ and $a$ are the vehicle velocity and acceleration respectively .

The present study is based on the World Motorcycle Test Cycle (WMTC), dedicated to low power motorcycles [10], and shown in Fig. 2.
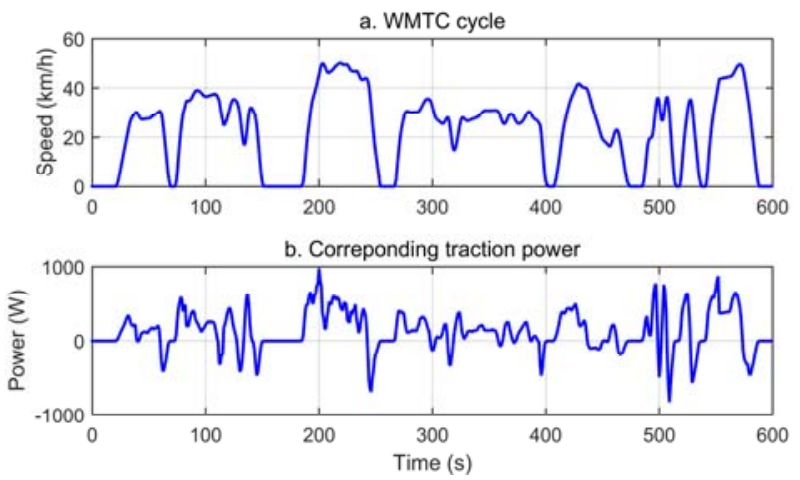

Fig. 2. WMTC driving cycle profile and corresponding traction power

\section{ENERGY MANAGEMENT STRATEGIES}

Optimal energy management is intended to determine the best instantaneous power split between the FC and the SC in order to minimize the fuel consumption for a given driving cycle. This can be handled as an optimal control problem, in which the control variable, denoted $u$, is the output FC power, the state variable, denoted $x$, is the energy stored in the SC, and the traction power is an external disturbance, denoted $w$.

The problem is defined by the set of equations (2) - (5), where $J$ is the total $H_{2}$ consumption over the considered time interval. Equation (3) models the dynamic behavior of the SC state of charge $x$ as a function of $u$ and $w$. Since the energy is provided solely by $\mathrm{H}_{2}$, the consumption is calculated with equal initial and final state for comparison sake. Finally, (5), (6) and (7) model the system operational constraints.

$$
\begin{gathered}
\text { Minimize } J=\int_{t_{0}}^{t_{f}} \dot{m}_{H_{2}}(u(t)) \cdot d t \\
\dot{x}(t)=f(u(t), x(t), w(t)) \\
x\left(t_{f}\right)=x\left(t_{0}\right)=x_{\text {ref }} \\
x_{\min } \leq x(t) \leq x_{\max } \\
\dot{x}_{\min } \leq \dot{x}(t) \leq \dot{x}_{\max } \\
u_{\min } \leq u(t) \leq u_{\max }
\end{gathered}
$$

In this work, two approaches proposed in literature are tested and compared. The first one is the adaptive equivalent consumption minimization strategy (A-ECMS) [7], whose main advantage is simplicity and low computation cost. The second one is stochastic dynamic programming (SDP) [8][9], which provides a clear mathematical background and a framework to include statistical information about the driving cycle. Online methods are sub-optimal and their results have to be compared to the off-line methods answers in order to rate their performance. In the present work, dynamic programming (DP) is used as benchmark, because it naturally accounts for the different operational constraints.

\section{A. A-ECMS}

A-ECMS is based on the concept of equivalence between the electric power provided by the storage unit $\dot{x}$ and some fuel consumption, obtained by applying an equivalent cost factor denoted $p$ hereafter. At all times, the optimal control should minimize a fuel consumption which includes the actual instantaneous hydrogen flow and the equivalent hydrogen consumption of the SC, as stated by (8).

$$
\forall t, u^{*}(x, w)=\underset{u}{\arg \min }\left[\dot{m}_{H_{2}}(u)+p \cdot \dot{x}(u, x, w)\right]
$$

The difficult point is to evaluate the equivalent cost $p$. In the case of off-line optimization, the problem is rigorously handled in the framework of variation calculus and Pontryaguin's minimum principle applies [3]. For online control, approaches are often empirical and are based on the fact that a small value of $p$ favors the use of the SC energy and tends to deplete it, whereas a large value favors the use of the $\mathrm{SC}$ energy and tends to charge the SC. Hence, $p$ can be used to control the SC state of charge $x$ in order to fulfill the constraints (4) and (5). In the present paper, the algorithm proposed in [7] is used, as interesting results are reported. The value of the equivalent cost is adjusted at regular intervals of time $T$, with a correction proportional to the difference between the current and reference $x$. A new value of $p$ is calculated for each period $[k T,(k+1) T]$ by using (9). This control tries to keep $x$ around the reference value.

$$
p_{k+1}=\frac{p_{k-1}+p_{k}}{2}+K_{p} \cdot\left(x_{r e f}-x(k T)\right)
$$

The parameters of the algorithm are the refreshment period $T$, the gain $K_{p}$ and the initial guesses $p_{0}$ and $p_{1}$. 
As formulated by (8) and (9), the A-ECMS cannot guarantee the respect of the operational state constraints (5). Therefore two additional heuristic rules are added to the original A-ECMS method. They are temporarily active when the instantaneous state gets close to its limits.

\section{B. Stochastic dynamic programing}

Stochastic dynamic programing (SDP) is a generalization of DP which provides a mathematical framework to account for random disturbances of the system. In the present case, the instantaneous traction power can be modeled as a random process, using statistical information about the forthcoming itinerary, available for example thanks to the navigation aid system.

Like DP, SDP requires the problem to be discretized in time and state. Let us denote respectively $0, k$ and $N$ the indexes of the initial, intermediate and final time steps. Let us also denote $x_{k}=x\left(t_{k}\right), u_{k}=u\left(t_{k}\right)$ and $w_{k}=w\left(t_{k}\right)$. At each time $t_{k}, w_{k}$ is a random variable characterized by a certain probability law. An average cost $J$ is defined by (10), where $E_{w_{k}}[\cdot]$ represents the mathematical expectation with respect to $w_{k}$ probability law. The discretized problem is given by (10)-(15).

$$
\begin{gathered}
\text { Minimize } J=\sum_{k=0}^{N-1} E_{w_{k}}\left[\dot{m}_{H_{2}}\left(u_{k}\right)\right] \cdot \Delta t \\
x_{k+1}=x_{k}+f\left(u_{k}, x_{k}, w_{k}\right) \cdot \Delta t \\
x_{0}=x_{N}=x_{r e f} \\
x_{\text {min }} \leq x_{k} \leq x_{\max } \\
\dot{x}_{\text {min }} \leq \dot{x}_{k} \leq \dot{x}_{\max } \\
u_{\text {min }} \leq u_{k} \leq u_{\max }
\end{gathered}
$$

As in DP, a so-called cost-to-go function, denoted $J_{k}(x)$, is defined at each time step $t_{k}$. It corresponds to the minimum average cost from a given state at time $t_{k}\left(x_{k}=x\right)$ to the final state $x_{N}$. This cost is calculated backwards, starting from the final step $N$ where an empirical penalty function favors $x_{N}$ close to $x_{r e f}$, and applying the recursive process (16)-(17) where $u_{k}{ }^{*}(x)$ denotes the optimal control at time $t_{k}$ as a function of the current system state $x_{k}=x$.

$$
\begin{aligned}
& J_{k}(x)=\min _{u}\left\{E_{w_{k}}\right. {\left[P_{\text {fuel }}(u)\right.} \\
&\left.\left.+J_{k+1}\left(x+f\left(u, w_{k}\right) \cdot \Delta t\right)\right]\right\} \\
& u_{k}^{*}(x)=\underset{u}{\operatorname{argmin}}\left\{E _ { w _ { k } } \left[P_{\text {fuel }}(u)\right.\right. \\
&\left.\left.+J_{k+1}\left(x+f\left(u, w_{k}\right) \cdot \Delta t\right)\right]\right\}
\end{aligned}
$$

At the end of the backward process, $J_{0}(x)$ represents the minimum fuel consumption which can be obtained starting from the initial state $x_{0}=x$. The optimal control policy $u^{*}=\left\{u_{k}{ }^{*}, 0 \leq k \leq N-1\right\}$ is then built by a forward process using the optimal control matrix calculated backwards $u_{k}^{*}(x)$. This forward process is applied to the online driving cycle, that is to say a given realization of the random process. The cost of a specific cycle may not be the lowest one, but the average cost of many cycles will be. DP corresponds to the particular case when $w_{k}$ is certain.
Accounting for the random nature of the driving cycle adds complexity and computational load, but this affects only the backward part of the algorithm, which is done off-line and only once. During the online process, only the forward part of the algorithm is applied to the actual driving cycle, and it basically consists in searching the command in the look-up table $u_{k}{ }^{*}(x)$, like in DP.

SDP provides an interesting framework, but the quality of the results relies on the quality of the random process model. In the present work, the driving cycle is modeled by a random speed characterized by a normal distribution $N(\mu, \sigma)$, as proposed in [9]. The parameters $\mu$ and $\sigma$ are calculated using all the points of the WMTC cycle.

\section{Simulation Calibration AND Assessment Results}

The two real-time EMS are evaluated in simulation. DP is used to provide reference results on the WMTC certification driving profile. Then, A-ECMS and SDP performances are evaluated, and their robustness is tested by changing the initial and final storage state of charge.

\section{A. Experimental calibration of the model and DP results}

The different EMS under study all rely on the system model to calculate at all times the best power split and minimize the $\mathrm{H}_{2}$ global consumption. Hence, it is crucial to first calibrate and validate this model and its setting parameters. In addition, the control part (low level and supervisor) also need to be validated. This is done using the test bench available at GeePs laboratory. After some simple tests not reported here, DP is used both to validate the model and control suitability, and to provide reference results, with respect to which the online EMS performances will be assessed.

The optimal FC power profile corresponding to WMTC is computed off-line by DP algorithm. This 10-min computed power profile is then used as the FC set point, while the load profile corresponds to WMTC. The resulting SC voltage trajectory is measured and converted into a SC energy profile. Fig 3 depicts the experiments and simulation behaviors, which are very close despite the large duration of the trial. This very good correlation between simulation and experiments results supports the model relevancy and validates the DP algorithm implementation.

In addition, Table I reports the average fuel consumption $J$ $\left[\mathrm{g} \cdot \mathrm{km}^{-1}\right]$, and the relative difference between the initial and final SC state $\Delta x[\%]$. The difference between the measured and calculated values is lower than $2 \%$ for the fuel consumption and $3 \%$ for the final state-difference. The similarity between these two overall performance indexes validates the accuracy of the FC/SC system model and the correctness of the EMS implementation.

TABLE I. OFF-LINE EMS : WMTC CYCLE RESULTS USING DP

\begin{tabular}{|l|l|l|l|l|}
\hline \multirow{2}{*}{ Strategy } & \multicolumn{2}{|c|}{$\mathbf{J}\left(\mathbf{1 0}^{-1} \mathbf{g} / \mathbf{k m}\right)$} & \multicolumn{2}{c|}{$\Delta \mathbf{x}(\%)$} \\
\cline { 2 - 5 } & \multicolumn{2}{|c|}{$\boldsymbol{s i m}$} & $\exp$ & \multicolumn{2}{|c|}{$\operatorname{sim}$} & $\exp$ \\
\hline DP & 3.70 & 3.74 & 0 & -2.5 \\
\hline
\end{tabular}



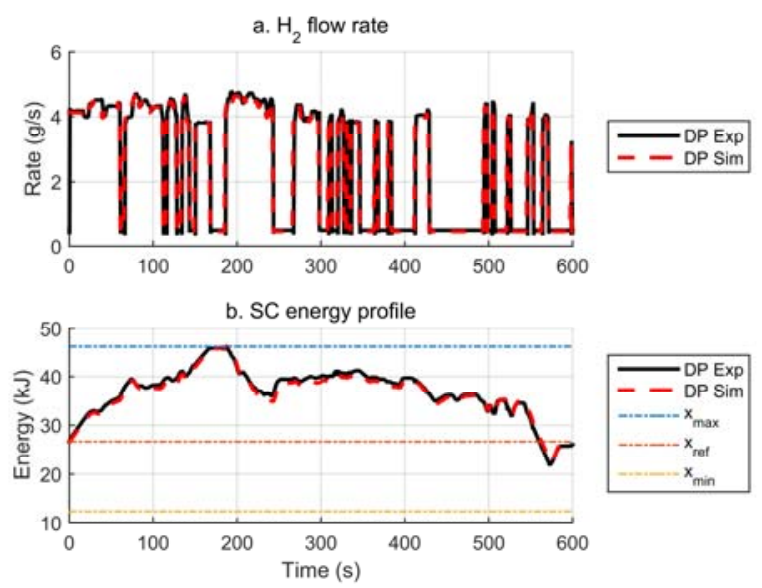

Fig. 3. Off-line EMS (DP): Comparison of system behaviour (instantaneous fuel consumption and SC energy profile)

\section{B. A-ECMS and SDP assessment under medium energy reference}

The aim of an EMS is to minimize the energy consumption, but also to ensure a desired final storage state of charge (SOC). Indeed, this latter point is crucial because, at the end of a first driving cycle, the EMS should ensure the possibility to start another mission under the best possible conditions: in this context, the initial state of charge is a key point. Therefore the present study adopts two evaluation criteria: first the global consumption $J$ and second the final state of charge error $\Delta x$ expressed as a percentage of the maximum SOC variation.

The first test to assess the online EMS performances is to determine their setting parameters using a given cycle (WMTC in the present case) and to compare their two evaluation criteria to DP. A significant element is the reference state of charge, which is settled at $x_{\text {ref }}=27 \mathrm{~kJ}$ which means a $44.1 \%$ relative state of charge. This value gives the EMS a great degree of freedom, possibly enabling deep SC state variations. Fig. 4 compares the SC state evolution $x(t)$ associated to the two real-time control strategies (A-ECMS and SDP) to the optimal one (DP).

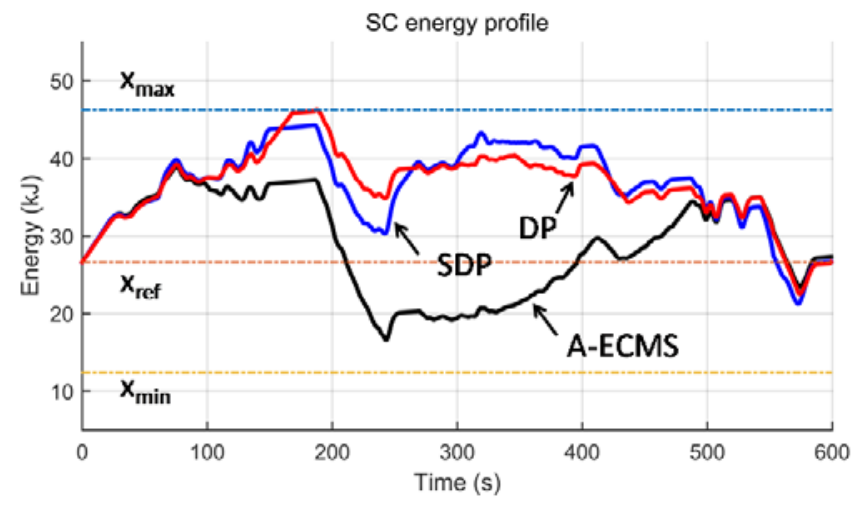

Fig. 4. Online EMS and DP simulation results : SC energy profile

A-ECMS tries to adjust online the equivalent cost factor $p$ to bring the system state back to its reference value $x_{\text {ref }}=27 \mathrm{~kJ}$. This behavior is consistent with this algorithm principle and it helps to explain why A-ECMS evolves around the state reference and is hence distant from the benchmark trajectory. Conversely, the SDP SC state behavior is much closer to DP's.

Table II reports the performance indexes of A-ECMS and SDP algorithms. Both obtain an overall fuel consumption close to the optimal one, but SDP brings the final state much closer to the reference value than A-ECMS does.

TABLE II. ONLINE EMS: WMTC CYCLE RESULTS

\begin{tabular}{|c|c|c|}
\hline \multirow{2}{*}{ Strategy } & $\mathbf{J ~}\left(\mathbf{1 0}^{-\mathbf{1}} \mathbf{g} / \mathbf{k m}\right)$ & $\Delta \mathbf{x} \mathbf{( \% )}$ \\
\cline { 2 - 3 } & $\operatorname{sim}$ & $\operatorname{sim}$ \\
\hline \multirow{2}{*}{ A-ECMS } & 3.76 & 2.6 \\
\hline \multirow{2}{*}{ SDP } & 3.72 & 0.2 \\
\hline
\end{tabular}

The control profiles found by the two online EMS are plotted in Fig.5. In both cases, the FC is operated mostly around $250 \mathrm{~W}$, which is its best efficiency.
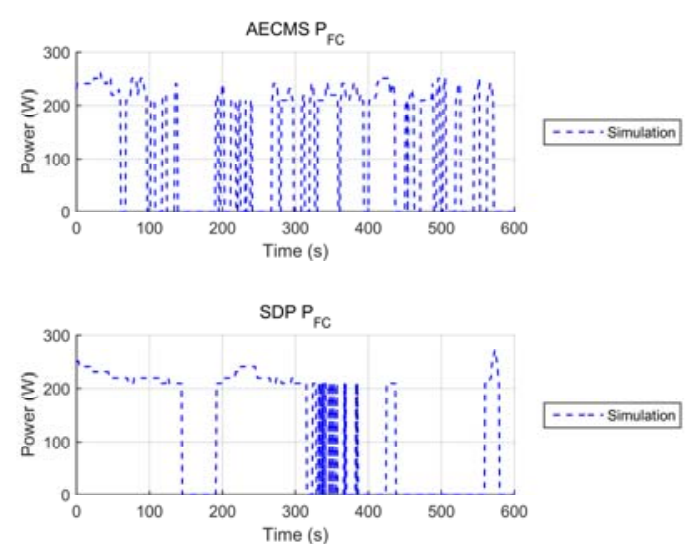

Fig. 5. Control profile comparaison of online strategies

\section{Sensitivity to the energy reference level}

The previous results are obtained for a medium reference state of $44.1 \%$ state of charge. In order to evaluate the impact of the reference state on the strategy performances, two different state reference values are tested: a low one of $20.6 \%$ $\left(x_{\text {ref,inf }}=19 \mathrm{~kJ}\right)$ and a high one of $73.5 \%\left(x_{\text {ref,sup }}=\right.$ $37 \mathrm{~kJ})$. To make data comparable, the driving cycle and the setting parameters of the EMS remain unchanged.

TABLE III. ONLINE EMS: WMTC CYCLE RESULTS

\begin{tabular}{|c|c|c|c|c|}
\hline \multirow{2}{*}{ Strategy } & \multicolumn{2}{|c|}{$\boldsymbol{x}_{\boldsymbol{r e f}}=\boldsymbol{x}_{\boldsymbol{r e f}, \boldsymbol{s u p}}$} & \multicolumn{2}{c|}{$\boldsymbol{x}_{\boldsymbol{r e f}, \boldsymbol{i n f}}$} \\
\cline { 2 - 5 } & $\boldsymbol{J}\left(\mathbf{1 0}^{-\mathbf{1}} \mathbf{g} / \mathbf{k m}\right)$ & $\boldsymbol{\Delta x}(\boldsymbol{\%})$ & $\boldsymbol{J}\left(\mathbf{1 0}^{-\mathbf{1}} \mathbf{g} / \mathbf{k m}\right)$ & $\Delta \boldsymbol{x}(\boldsymbol{\%})$ \\
\hline A-ECMS & 3.76 & 3.4 & 4.35 & 6.0 \\
\hline SDP & 3.68 & -1.5 & 3.77 & 1.9 \\
\hline DP & 3.67 & 0 & 3.73 & 0 \\
\hline
\end{tabular}

Table III reports the fuel consumption $J$ and the difference between the reference and final SC state $\Delta x$ for the two given energy references. It permits to assess that this parameter has a great impact on the A-ECMS consumption. For instance, in the low reference state, the consumption is $16.6 \%$ above the 
benchmark consumption. Similarly, the final state accuracy is also affected by the reference change.

Conversely, SDP results show a good consumption performance, very close from the benchmark one. Moreover, the final state is also quite close to the reference one. From those results, it can be concluded that the reference state value has little effect on SDP performances.

Fig. 6 and Fig. 7 show the SC energy profiles obtained respectively with the low and high reference state. It can be seen that the operational constraint on the SC state of charge operates. DP and SDP naturally account for it, whereas AECMS rely on heuristics which probably degrades the method performance. Furthermore, the very principle of A-ECMS consists in maintaining the storage energy level close to the reference one, which may be far from the optimal trajectory .

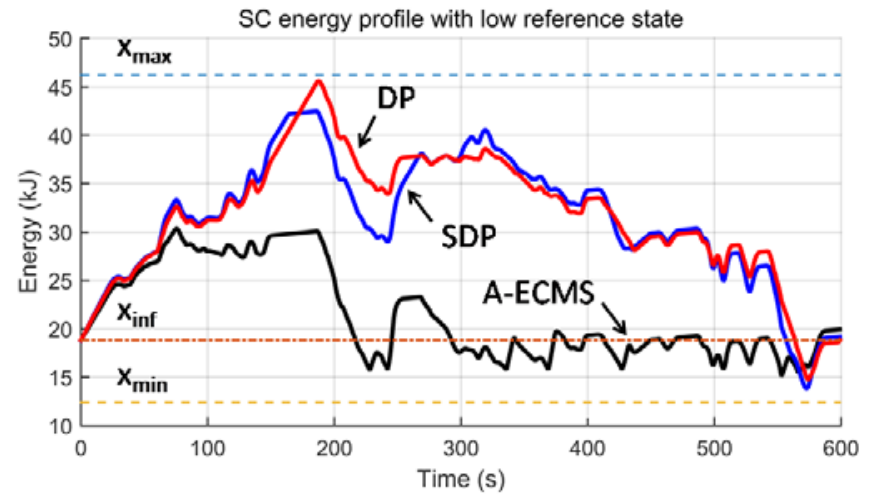

Fig. 6. SC energy profiles using online EMS with low reference state

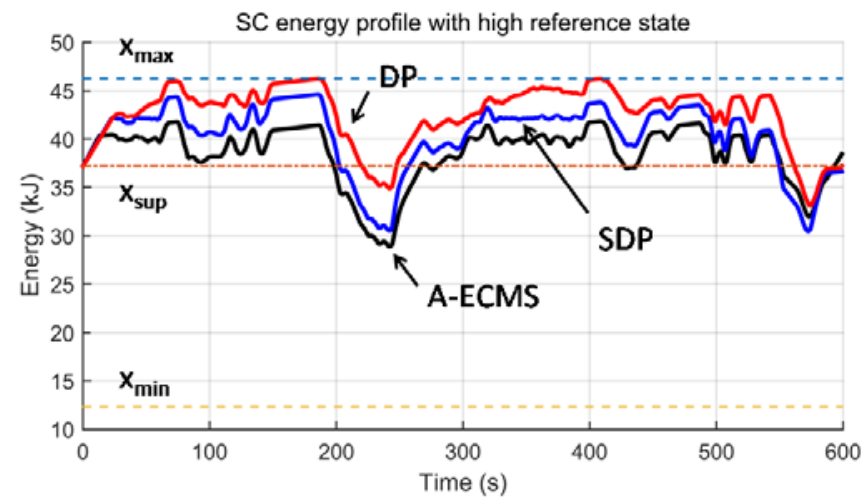

Fig. 7. SC energy profiles using online EMS with high reference state

\section{CONCLUSION}

Powering an electric scooter with a FC is an attractive alternative to battery supply in terms of autonomy and speed of refueling. This solution requires the use of an additional storage element such as a SC. This gives the possibility to implement an effective and robust EMS which achieves at any time an instantaneous power split as close as possible to the optimal one. This study is carried out by simulation.
For this purpose, the system under study is carefully modeled and its behavior is successfully validated using a laboratory test bench. In addition a standard online EMS, namely DP, is considered as a benchmark; its proper functioning is also positively tested.

In this research, the aim is to evaluate the potential of two promising online energy management methods using a single driving profile on which the setting parameters of both approaches are tuned. Simulation and experiments results show that SDP has a higher potential than A-ECMS. Indeed, using a standard medium storage reference, A-ECMS performs somewhat less well than the target DP: its dihydrogen consumption is slightly higher and its final state is a little away from the reference state. Moreover, A-ECMS is very sensitive to the storage reference state. Its performance is severely degraded when the reference value moves away from the mid-point of the state of charge range. For instance, and compared to the benchmark, the dihydrogen consumption can grow by more than $16 \%$ in a case of low reference state. Conversely, SDP sub-optimal behavior remains close to the benchmark answer; in the global study, its consumption does not exceed $1 \%$ of the DP's one. In conclusion, it makes SDP complexity worth handling.

This study should be continued and extended. The base line for further work is to validate the good simulation results on the test bench and to consider a set of random driving cycles so as to assess the in line EMS real-time performances.

\section{REFERENCES}

[1] N. SamExp, Fuel cell technology: reaching towards commercialization, Springer Science \& Business Media, 2006.

[2] Q. Li, W. Chen, Z. Liu, M. Li and L. Ma, "Development of energy management system based on a power sharing strategy for a fuel cellbattery-supercapacitor hybrid tramway”, J. Power Sources. vol.279, pp. 267-280, 2015.

[3] D.E. Kirk, Optimal control theory an introduction, Dover Publications, 2012

[4] N. Kim, S. Cha and H. Peng, "Optimal Control of Hybrid Electric Vehicles Based on Pontryagin's Minimum Principle”, IEEE Trans. Control Syst. Technol. vol. 19, pp. 1279-1287, 2011.

[5] D.P. Bertsekas, Dynamic programming and optimal control, 3rd edition, Athena Scientific, 2005.

[6] M. Ansarey, M.S. Panahi, H. Ziarati and M. Mahjoob, "Optimal energy management in a dual-storage fuel-cell hybrid vehicle using multidimensional dynamic programming”, J. Power Sources. vol. 250, pp. 359-371, 2014.

[7] S. Onori, L. Serrao and G. Rizzoni, "Adaptive equivalent consumption minimization strategy for hybrid electric vehicles", ASME 2010 Dyn. Syst. Control Conf., pp. 499-505, 2010.

[8] X. Jiao and T. Shen, "SDP policy iteration-based energy management strategy using traffic information for commuter hybrid electric vehicles", Energies, vol. 7, no. 7, pp. 4648-4675, 2014.

[9] J. Liu and H. Peng, "Modeling and control of a power-split hybrid vehicle”, IEEE Trans. on Control Syst. Technol., vol.16, no. 6, pp. 12421251, 2008.

[10] T.J. Barlow, S. Latham, I.S. McCrae and P.G. Boulter, A reference book of driving cycles for use in the measurement of road vehicle emissions, TRL Publ. Proj. Rep., 2009. 